\title{
Correction to: Surgical management of choroid plexus papilloma of the cerebellopontine and cerebellomedullary angle: classification and strategy
}

\author{
S. D. Adib ${ }^{1} \cdot$ J. M. Hempel ${ }^{2} \cdot$ K. Kandilaris $^{3} \cdot$ F. Grimm ${ }^{1} \cdot$ R. Evangelista Zamora $^{1} \cdot$ M. Tatagiba ${ }^{1}$ \\ Published online: 12 April 2021 \\ ๑) Springer-Verlag GmbH Germany, part of Springer Nature 2021
}

\section{Correction to: Neurosurg Rev \\ https://doi.org/10.1007/s10143-021-01506-4}

The authors regret that there was a mistake in one of the sentences in the original article.

In the section "Results" in the subsection "Histopathological results" the authors wrote in the first sentence:

"CPP was histologically confirmed in each case (Table 1) with typical characteristics such as a strong immunoreacticity for SYN, CK, S-100 protein, and negative immunoreactivity for epithelial membrane antigen and glial fibrillary acidic protein, and no or low mitotic activity."

The online version of the original article can be found at https:// doi.org/10.1007/s10143-021-01506-4

\section{S. D. Adib}

sasan_adib2002@yahoo.de

J. M. Hempel

Johann-martin.hempel@med.uni-tuebingen.de

K. Kandilaris

Kosmas.kandilaris@med.uni-tuebingen.de

F. Grimm

florian.grimm@med.uni-tuebingen.de

R. Evangelista Zamora

rocio.evangelista@med.uni-tuebingen.de

M. Tatagiba

marcos.tatagiba@med.uni-tuebingen.de

1 Department of Neurosurgery, University of Tuebingen, Hoppe-Seyler-Str. 3, 72076 Tuebingen, Germany

2 Department of Neuroradiology, University of Tuebingen, Hoppe-Seyler-Str. 3, 72076 Tuebingen, Germany

3 Department of Neuropathology, University of Tuebingen, Hoppe-Seyler-Str. 3, 72076 Tuebingen, Germany when it should have been:

"CPP was histologically confirmed in each case (Table 1) with typical characteristics such as papillary architecture, membranous staining for Kir7.1; and no or low mitotic activity."

The original article has been corrected.

Publisher's note Springer Nature remains neutral with regard to jurisdictional claims in published maps and institutional affiliations. 